\title{
LA PROBLEMÁTICA DEL MANTENIMIENTO EN LOS RELLENOS DE LA LÍNEA DE ALTA VELOCIDAD MADRID-SEVILLA
}

\author{
(MAINTENANCE PROBLEMS WITH THE FILLING ON THE HIGH SPEED RAIL LINE)
}

Julio Barcones Mingueza, Ingeniero de Caminos

Jefe de Infraestructura de AVE

Eduardo Pradera, Ingeniero de Caminos

ICYFSA

\section{RESUMEN}

El comportamiento de los rellenos en una línea de Alta Velocidad es importante, tanto desde el punto de vista de la comodidad del viajero, como de la seguridad de rodadura de los vehículos ferroviarios; de ahi, la importancia del seguimiento de su evolución tanto, durante la fase constructiva, como en la posconstructiva. El presente artículo trata de la sistemática de control establecida para este tema en la línea de AVE.

\section{SUMMARY}

The performance of the filling on a high speed line is important, not only for the traveler's comfort but also for the safety of the treads of the train. Hence, the importance of following its evolution, not only during the construction phase but in the post-construction phase as well. This article deals with the control system established for such a follow-up on the high speed line.

\section{INTRODUCCIÓN}

El conjunto de las obras incluidas comúnmente bajo la denominación genérica de Movimiento de Tierras representa en cualquier línea ferroviaria más del $90 \%$ de su longitud, distribuyéndose sensiblemente por igual entre rellenos y desmontes.

Como consecuencia, el comportamiento de este tipo de obras durante la explotación tiene una importancia fundamental en cuanto a la incidencia de las labores de mantenimiento, representando un gran porcentaje de las que, de forma continua, hay que desarrollar a lo largo de la vida de la obra.
Centrándonos en la problemática de los rellenos, la casuística de las incidencias posibles tiene casi siempre una relación directa con la deformación de los mismos, si como consecuencia de la misma se produce la modificación de la geometría de la rodadura en una magnitud tal que llegue a afectar a la confortabilidad $o$, incluso, a la seguridad del tráfico.

No obstante, aun siendo conceptualmente la deformación el único objeto de preocupación, cara a la conservación, es preciso prevenir las múltiples causas que pueden llegar a producirla y que por tanto han de ser objeto de una vigilancia aplicada con intensidad adecuada a la importancia del riesgo que puede llegar a represen- 
tar, lo que es función del binomio: magnitud del defecto y características de la línea.

Los procesos de deformación que pueden llegar a presentarse en los rellenos pueden dividirse en tres grandes grupos:

- Procesos de consolidación natural.

- Procesos de desestabilización.

- Procesos erosivos.

Los procesos de consolidación natural se producen en las obras de reciente construcción y se manifiestan en el asiento progresivo de la masa del relleno y de su cimiento. Este asiento es proporcional a la altura del relleno en cada punto y se produce, en condiciones normales, sin un cambio significativo en el estado tensional alcanzado al final de la construcción, por lo que no suelen llegar a producir fenómenos de inestabilidad.

Por el contrario los procesos de desestabilización, con mayor o menor extensión, suponen la rotura del material de relleno lo que puede producirse por un cambio en el estado tensional de la masa, por una disminución de las características resistentes del material, o por la conjunción de ambos factores.

Por último se pueden presentar procesos erosivos, generalmente de carácter superficial y puntual, los cuales tienen su origen en el arrastre de material que produce la escorrentía de las aguas de lluvia y que puede llegar a afectar a la plataforma, bien de forma directa por socavación del apoyo de las traviesas o indirectamente provocando deslizamientos de tipo local por la modificación que las cárcavas introducen en la geometría de los taludes.

El mantenimiento de cualquier línea ferroviaria ha de dar solución a los distintos problemas anteriormente citados, debiendo hacerlo además de una forma adecuada a las características de la línea de que se trate.

En el caso de la Línea del AVE entre Madrid y Sevilla se dan multitud de circunstancias específicas que hacen necesario prestar una especial atención a la problemática del mantenimiento general de las obras de infraestructura y especialmente a la de los rellenos. La respuesta a estas singularidades se ha hecho aplicando diversas medidas en todas y cada una de las distintas fases que se desarrollan a lo largo del proceso de construcción de una obra de este tipo, cualquiera que sea su envergadu(c) Consejo Superior de Investigaciones Científicas Licencia Creative Commons 3.0 España (by-nc)

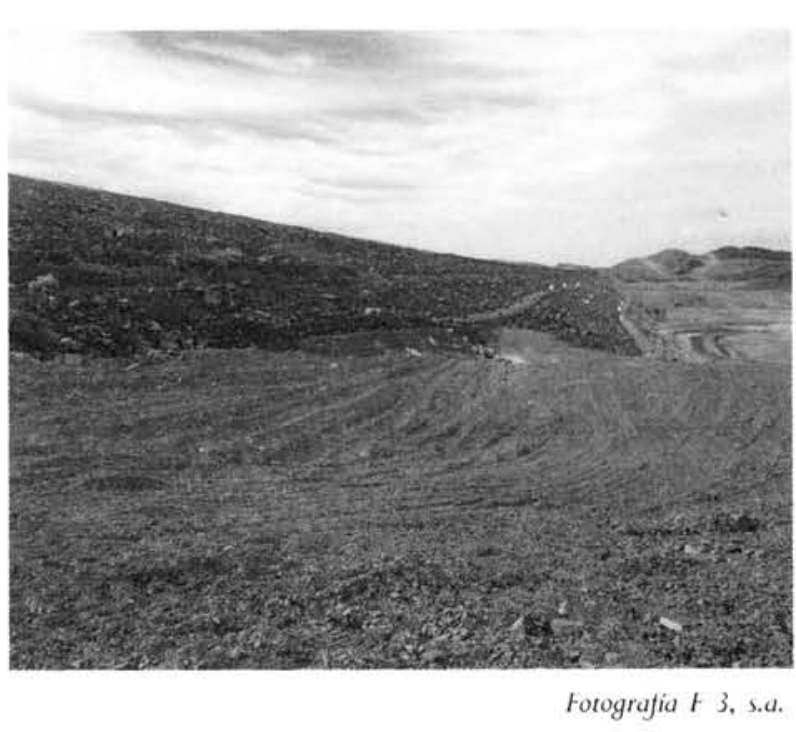

Foto 1.-Zona de rellenos en el tramo Brazatortas-Córdoba.

ra, es decir, desde la concepción del proyecto hasta la planificación y ejecución del mantenimiento, pasando por la propia labor de construcción.

El destino de la línea al tráfico ferroviario en Alta Velocidad precisa de la adopción de amplios parámetros de trazado, lo que da como resultado la magnificación de las dimensiones de las obras en general y particularmente de los rellenos, especialmente cuando la topografía es abrupta como en nuestro caso sucede en las zonas de paso de las distintas sierras que cruza la línea y de forma muy particular en la de Sierra Morena.

Por otra parte el alto nivel de confort y seguridad que exige el tipo de tráfico a que se destina, requiere una especial rigurosidad en el mantenimiento de las condiciones geométricas de la vía.

La conjunción de ambas características da como resultado que sea necesario prestar una especial atención a la evolución del comportamiento de los rellenos a lo largo de los aproximadamente $220 \mathrm{~km}$ del trazado de la línea Ferroviaria de Alta Velocidad entre Madrid y Sevilla, que está soportada por este tipo de elementos. (Foto 1).

Consecuentemente se ha planteado en esta línea una actuación de mantenimiento que puede considerarse pionera en nuestro país, tanto en ámbito ferroviario como en el de otras obras de infraestructura del transporte, no sólo cuantitativamente por la magnitud de las obras sobre las que se actúa, sino también en el aspecto cualitativo por el carácter eminentemente previsor que le conhttp://informesdelaconstruccion.revistas.csic.es 
fiere la realización de un sistemático seguimiento técnico del comportamiento y evolución de las obras. Tal planteamiento, además de la evidente utilidad que representa cara a la conservación de la línea, potenciando la corrección de las causas frente a la de los efectos, permitirá la mejora de los criterios de diseño bajo los cuales habrán de proyectarse las futuras líneas de Alta Velocidad de nuestro país, trasladando a ellos las experiencias obtenidas.

\section{CRITERIOS DE PROYECTO Y CONSTRUCCIÓN}

Los altos niveles de calidad de la rodadura exigidos en la línea Madrid-Sevilla harían inviable un adecuado mantenimiento de la misma si no se hubieran adoptado en los proyectos las medidas necesarias para limitar, hasta niveles aceptables, los inevitables asientos que a lo largo del tiempo se producen en los rellenos. Este condicionamiento se puede decir que ha constituido la preocupación fundamental en la concepción del diseño de los rellenos, más allá de la solución estricta de la estabilidad de los mismos.

No nos extenderemos sobre la problemática de la estabilidad de los rellenos por constituir una cuestión geotécnica suficientemente conocida, la cual ha sido convenientemente solucionada en fases de proyecto y de construcción, mediante el oportuno diseño de la geometría y las necesarias prescipciones concernientes a las características de los materiales y de su puesta en obra. Tanto la labor de investigación geotécnica complementaria como la de definición constructiva de los rellenos se ha extendido durante la ejecución, lo que ha permitido mejorar los proyectos iniciales a partir del más profundo conocimiento de los terrenos que facilita el propio proceso constructivo.

La gran longitud de la línea y la diversidad geotécnica y morfológica de los terrenos afectados ha dado como resultado una considerable variedad de soluciones en el diseño de los rellenos, lo que en muchos ha sido consecuencia de la adaptación a las características reales puestas de manifiesto durante la construcción.

De esta manera se han adoptado a lo largo del trazado taludes con inclinaciones variables entre el 1,5 (H) / 1 (V) y el 2(H) / $1(\mathrm{~V})$, disponiéndose, en ocasiones, bermas de 4 a 5 metros de anchura, en número variable según la altura total del talud.

En cuanto a las condiciones de puesta en obra también ha sido necesario adoptar distintas prescripciones según la problemática de los materiales, exigiéndose, según los casos, densidades de compactación que han oscilado entre el 95\% del Próctor Normal y del Modificado, así como la aplicación de procedimientos y maquinaria especiales para conseguir una sustancial disminución en el índice de huecos del producto final.

Como ya se ha comentado, el objetivo del diseño finalmente adoptado en cada caso no ha sido exclusivamente el de asegurar la estabilidad de los rellenos frente a la rotura, lo que ha resultado ampliamente garantizado, sino también el de limitar el valor de la consolidación postconstructiva que ha de producirse durante los primeros años de vida de la obra, lo cual resulta, evidentemente, un criterio mucho más restrictivo.

A pesar de estas medidas dicha consolidación da lugar a una especial problemática en las zonas de transición entre los rellenos y cualquier otro elemento con un comportamiento más rígido, debido al asiento diferencial que se produce entre secciones transversales inmediatas, en las que existen alturas de relleno muy diferentes.

Tal problemática tiene lugar de forma fundamental en la transición de los estribos en los viaductos y puentes, así como en el paso sobre obras de fábrica, dependiendo la importancia del problema de la altura de la obra de hormigón y, en su caso, de la profundidad de la misma respecto de la plataforma.

Análogamente, en los tramos en los que el terreno natural presenta una fuerte inclinación, puede darse un fenómeno similar en las zonas de transición entre relleno y desmonte.

Para la solución de este tipo de problema en las obras de hormigón, además de prescribirse la limitación de la altura de los estribos de los viaductos a un valor máximo de 15 metros, ha sido necesario proyectar diversos tipos de obras denominadas "cuñas de transición", siendo la tipología más significativa la que se representa en las figuras 1,2 y 3 . Estas obras producen una graduación longitudinal en la deformabilidad total de las sucesivas secciones. 


\section{CUÑAS DE TRANSICIÓN EN OBRAS CIRCULARES ENTERRADAS}
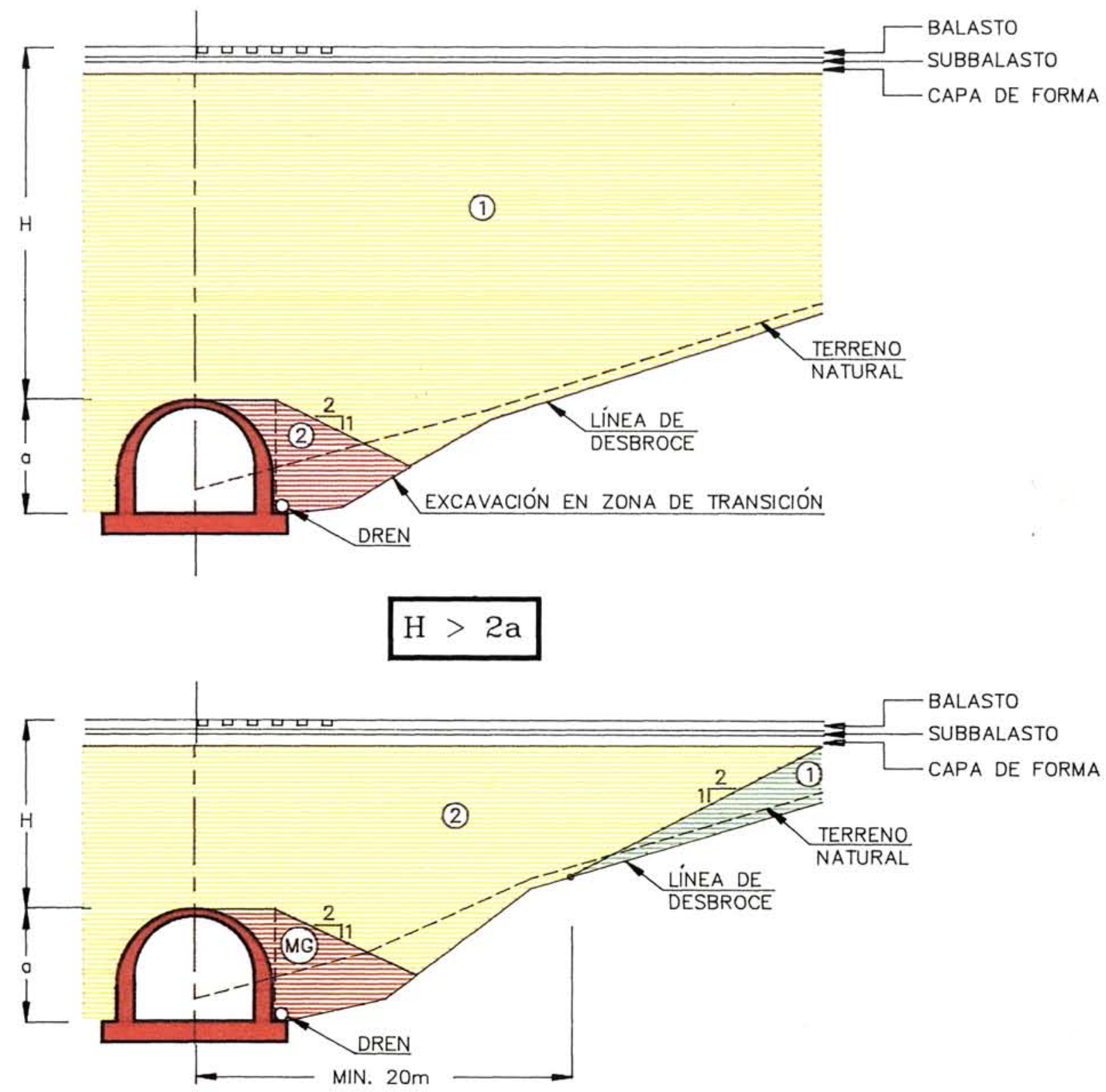

$2 \mathrm{a}>\mathrm{H}>\mathrm{a}$

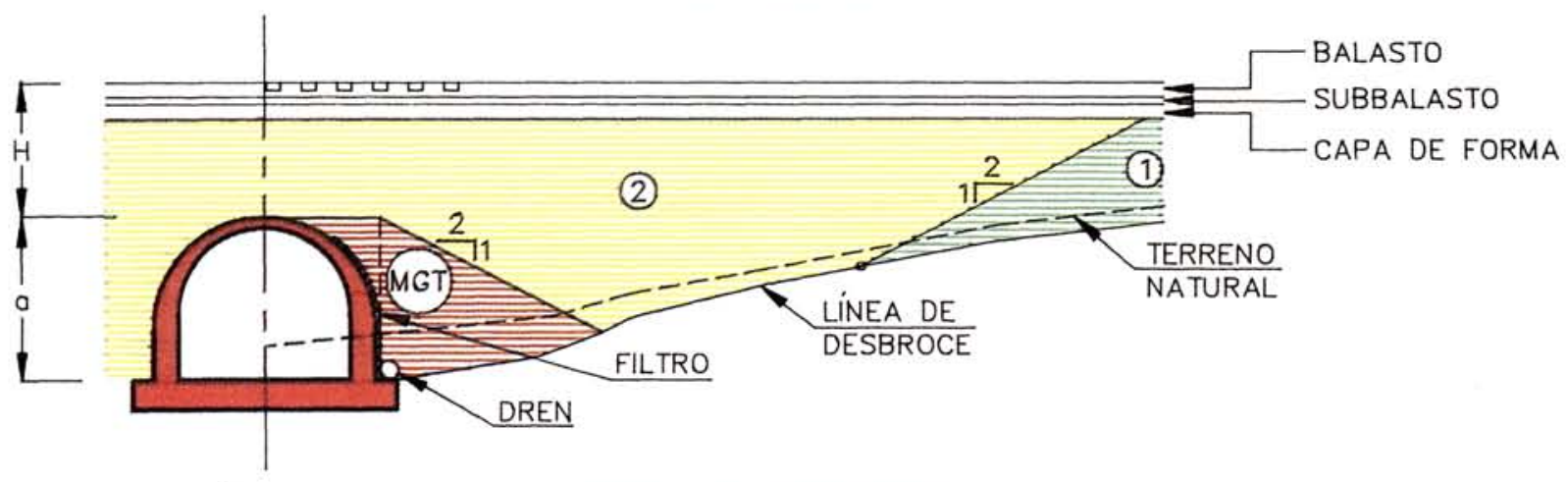




\section{CUÑAS DE TRANSICIÓN EN OBRAS ENTERRADAS PLANAS}

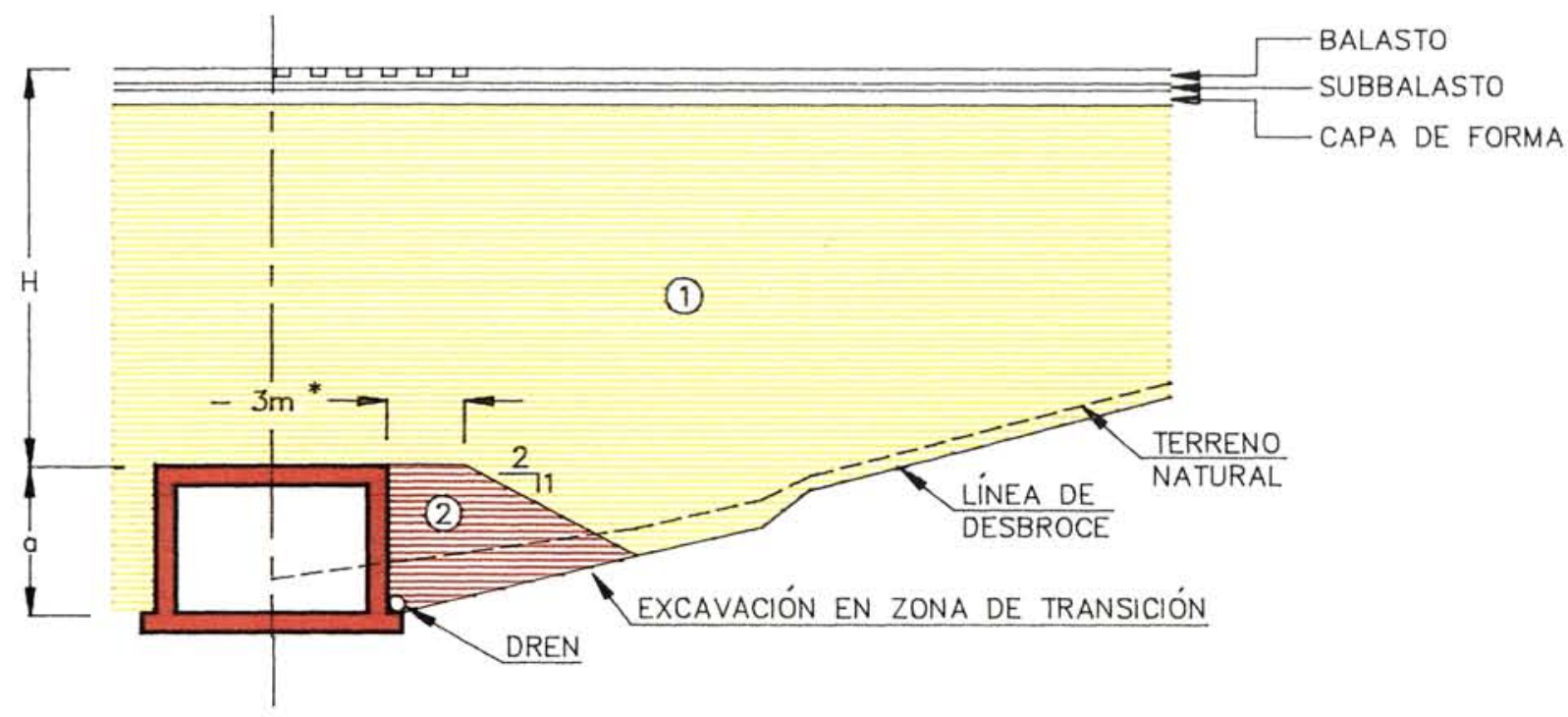

$\mathrm{H}>2 \mathrm{a}$

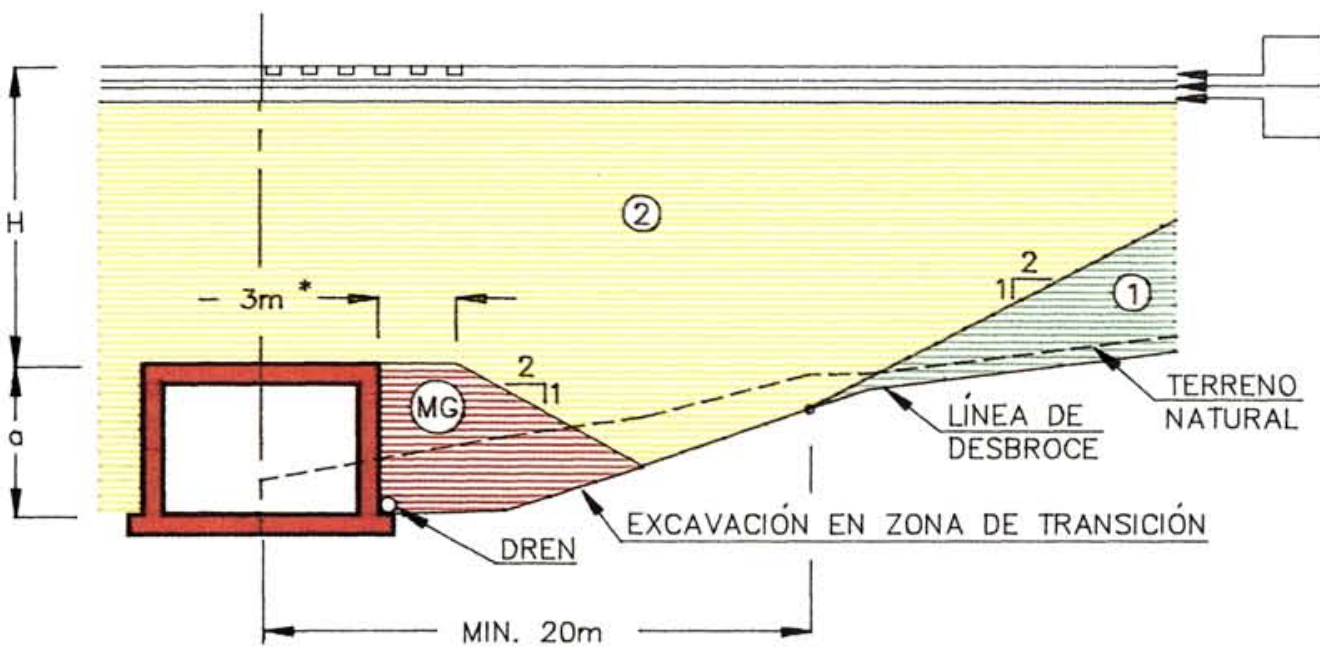

$$
2 \mathrm{a}>\mathrm{H}>\mathrm{a}
$$

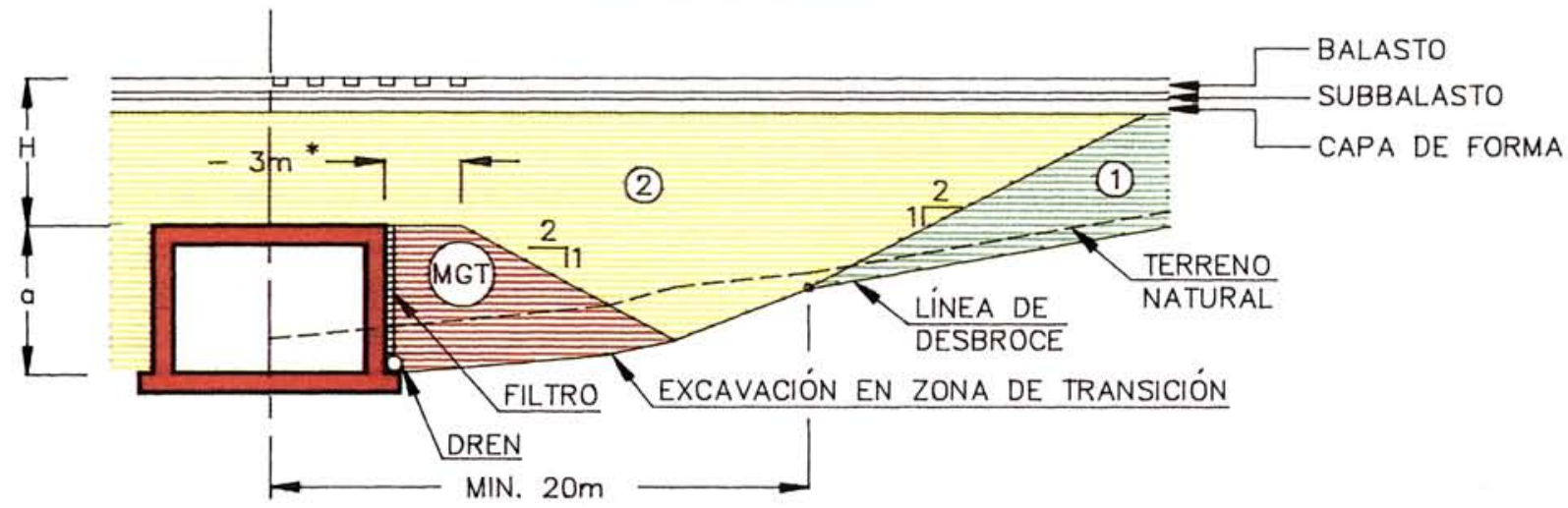




\section{CUÑAS DE TRANSICIÓN EN ESTRUCTURAS SUPERFICIALES}

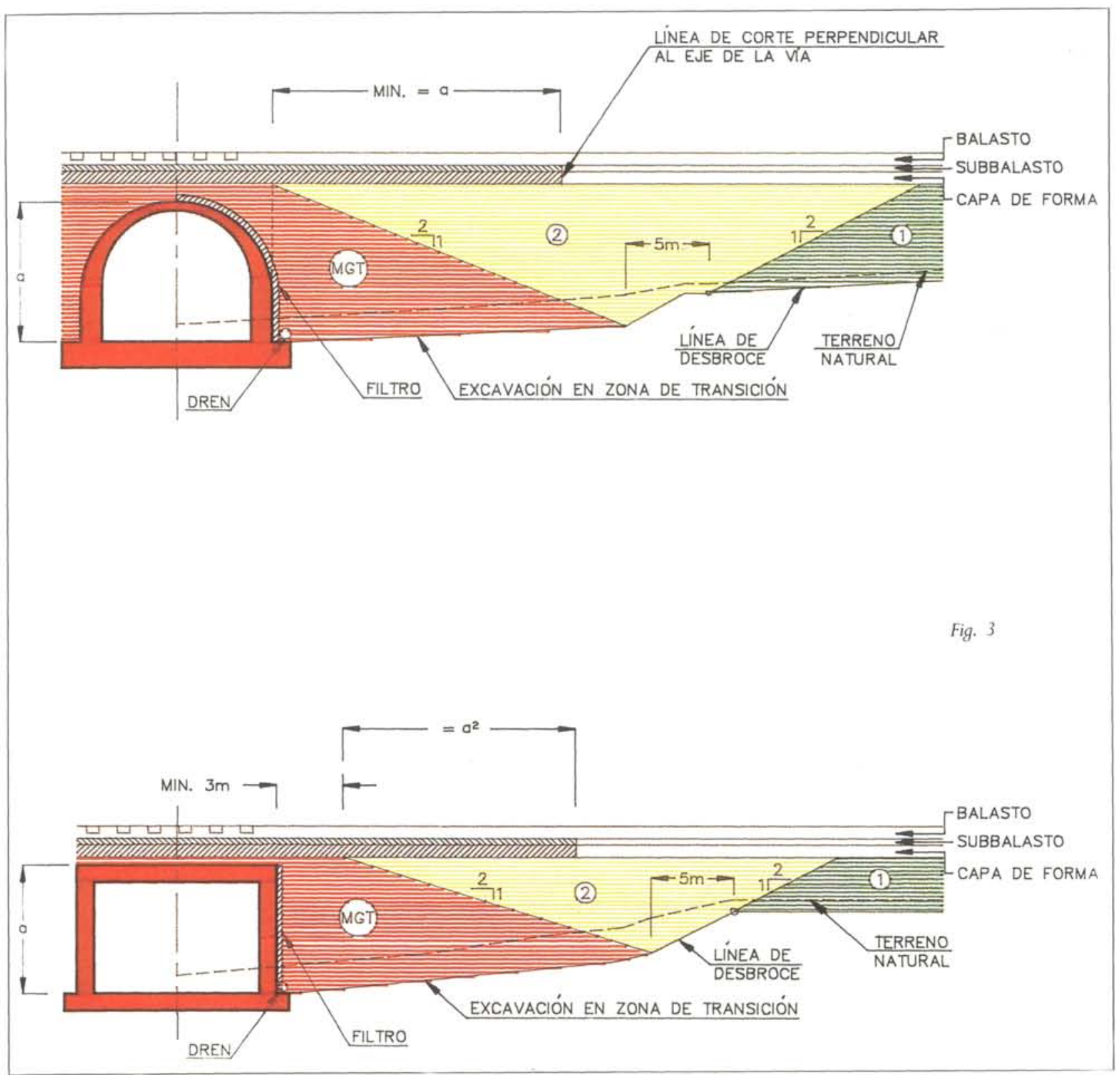

El criterio adoptado para la definición geométrica de estas transiciones ha sido el de limitar la deformada de la plataforma que pudiera llegar a producirse por el asentamiento diferencial, a un radio de curvatura mínimo de $50 \mathrm{~km}$, lo que equivale a una prescripción de $10 \mathrm{~cm}$ en $30 \mathrm{~m}$.

Los distintos materiales que se incluyen en la formación de las cuñas, y que se identifican en los esquemas de las figuras citadas, han sido:

1 - Relleno General.

(c) Consejo Superior de Investigaciones Científicas Licencia Creative Commons 3.0 España (by-nc)
2 - Relleno Seleccionado.

MG - Material Granular.

MGT - Material granular tratado con 3\% de cemento.

En las transiciones sobre obras superficiales se incorpora, además de la cuña correspondiente, el tratamiento con cemento de las capas de subbalasto y de la capa de forma, en una longitud igual a la altura de la obra de fábrica.

Las características de los materiales empleados en la formación de las cuñas son las que se indican en el cuadro 1. http://informesdelaconstruccion.revistas.csic.es 


\section{TRANSICIÓN RELLENO-DESMONTE}

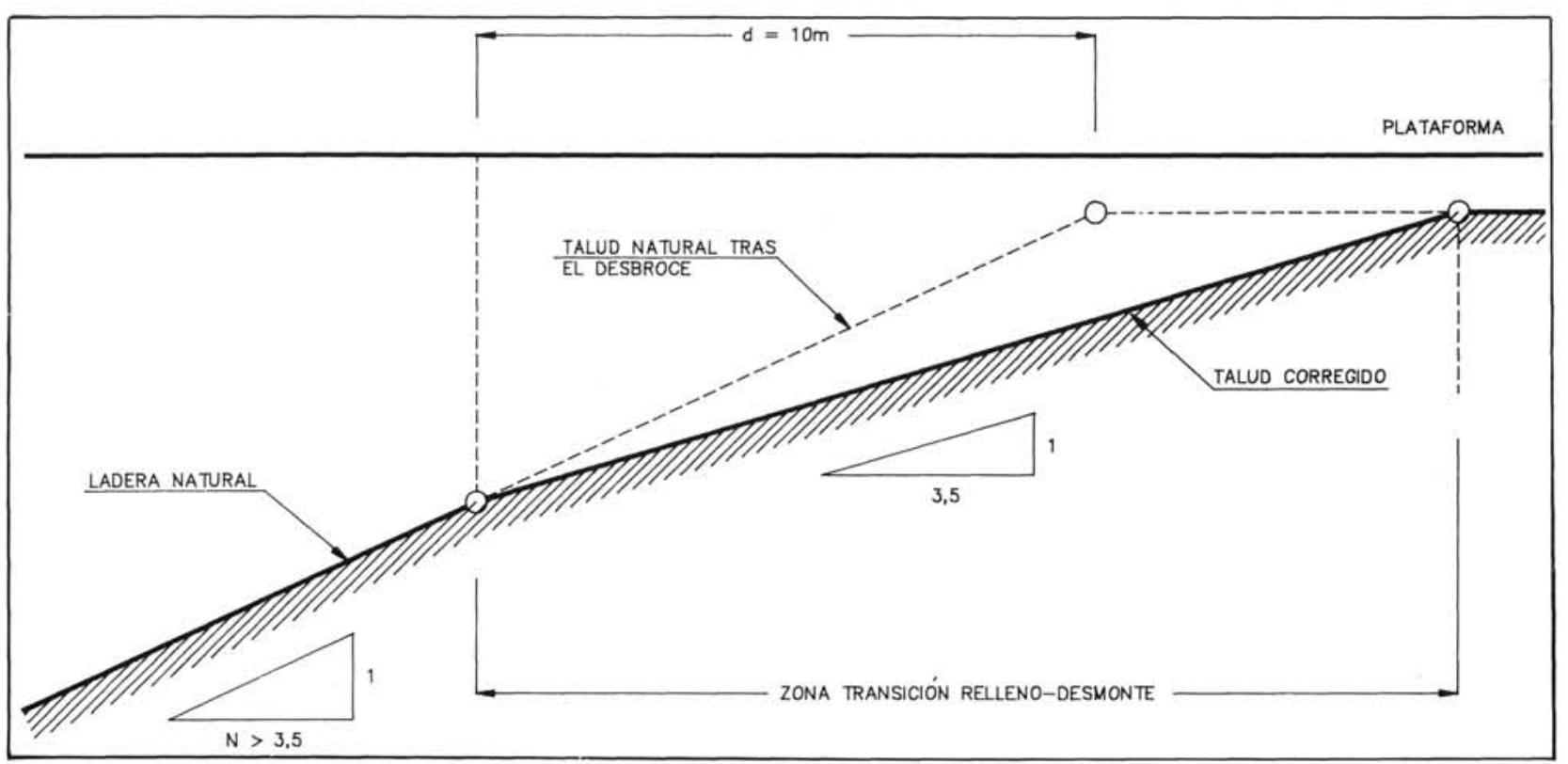

Fig. 4

\section{CUADRO 1}

\begin{tabular}{|l|c|c|}
\hline \multicolumn{3}{|c|}{ CARACTERÍSTICAS DE IDENTIFICACIÓN } \\
\hline Tamaño máximo & 10 a $15 \mathrm{~cm}$ & $5 \mathrm{~cm}$ \\
\hline Huso granulométrico & NO & SI \\
\hline$\%$ Finos & $<40 \%$ & $<5 \%$ \\
\hline Equivalente de Arena & & $>30$ \\
\hline Límite Liquido & $<35$ & No Plástico \\
\hline Densidad Próctor Normal & $>1,75 \mathrm{~kg} / \mathrm{dm}^{3}$ & \\
\hline CBR & $>5$ & \\
\hline Hinchamiento en CBR & $<2 \%$ & \\
\hline Desgaste de Los Ángeles & \multicolumn{3}{|c|}{$<$} \\
\hline \multicolumn{2}{|c|}{ ESPECIFICACIONES DE PUESTA EN OBRA } \\
\hline Espesor de Tongada & $<40 \mathrm{~cm}^{2}$ & \\
\hline Densidad Mínima & $>95 \%$ PM & $>95 \%$ PM \\
\hline Placa de Carga (2. ciclo) & $450-600 \mathrm{~kg} / \mathrm{cm}^{2}$ & \\
\hline
\end{tabular}

Nota. $\mathrm{Al}$ relleno seleccionado se le exige, adicionalmente, un módulo mayor de $500 \mathrm{~kg} / \mathrm{cm}^{2}$ en el primer ciclo de la placa de carga.

La caracterización de la deformabilidad postconstructiva de los materiales no tratados con cemento se ha realizado de acuerdo con el criterio, deducido de las experiencias publicadas sobre esta cuestión, de que la mayor parte (c) Consejo Superior de Investigaciones Cientificas Licencia Creative Commons 3.0 España (by-nc) de los asientos de este tipo se desarrollan a lo largo de los primeros 10 años siguiendo una ley de tipo logarítmico, y que su magnitud total es un $20 \%$ de la deformación constructiva, produciéndose la mitad del mismo durante el primer año y el resto durante los nueve siguientes.

En cuanto a las transiciones relleno-desmonte se ha solucionado mediante la suavización geométrica del terreno natural hasta conseguir un ángulo de $16^{\circ}$ cuando la ladera supera esta inclinación. El punto de arranque de esta suavización se sitúa en la zona de relleno y a una distancia de $10 \mathrm{~m}$ del de contacto de la subrasante, como se puede ver en el esquema ilustrativo que se acompaña (Fig. 4).

\section{CONTROL DURANTE EL MANTENIMIENTO}

Durante los primeros años de explotación de la línea se ha previsto el seguimiento técnico de la evolución de los rellenos y cuñas de transición, lo que permitirá comprobar el adecuado comportamiento de los mismos así como el cumplimiento de las hipótesis fundamentales de todo tipo que se establecieron para ellos en la fase de proyectc. 


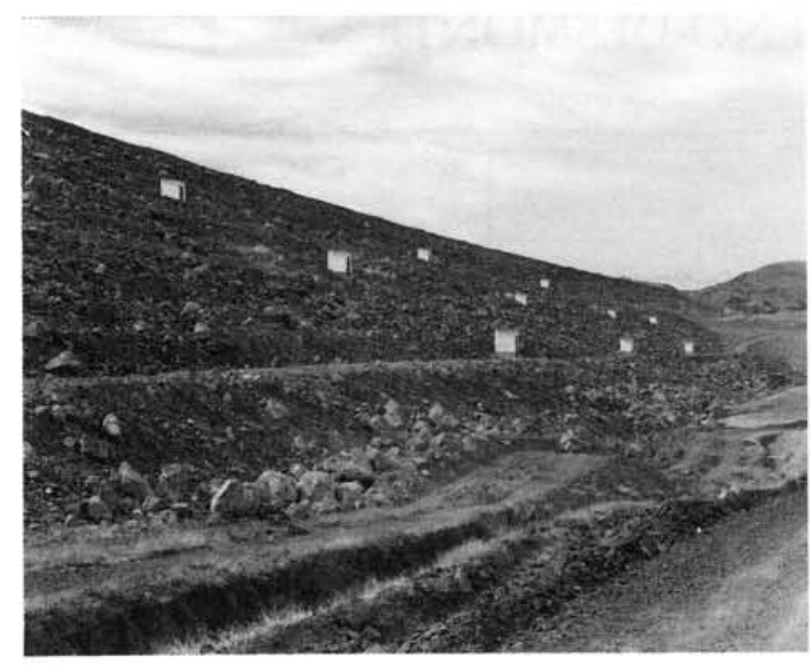

Fotografia F 3, s.a.

Foto 2.-Distribución de células hidráulicas en una sección de relleno.

En cuanto a las condiciones de estabilidad de los taludes la labor se ha planteado, inicialmente, en base a la vigilancia control del mantenimiento de la funcionalidad de las obras de drenaje transversal y de las cunetas de guarda, ya que constituyen los elementos que garantizan la continuidad en el tiempo de las condiciones de trabajo de los rellenos, tanto en cuanto a las hipótesis de carga como en lo relativo a los estados tensionales y a las características resistentes de los materiales. Este tipo de actuación, unido a los recorridos sistemáticos de inspección visual dirigidos para detectar la aparición de síntomas de movimientos en las cabezas y zonas de cimiento, constituyen la base del seguimiento de la evolución de las condiciones de estabilidad alcanzadas durante la construcción.

En cuanto al comportamiento deformacional el seguimiento visual anteriormente citado se complementa con el registro de los movimientos de asentamiento, para lo cual se utilizan los mismos sistemas de instrumentación que se colocaron durante la construcción en 21 rellenos distribuidos a lo largo de la línea, los cuales fueron seleccionados, en su día, por su representatividad dentro de la casuística global del conjunto de los que se incluyen en ella.

La instrumentación de dichos rellenos ha consistido en la colocación de una serie de células hidráulicas dispuestas en 1, 2 ó 3 secciones transversales, dependiendo de la magnitud del elemento. En cada sección se dispone nor- malmente de tres niveles, con un número de células por nivel que varía entre 3 y 5 unidades, siendo la distribución típica la que se indica en la foto 2 .

En fase postconstructiva dicha instrumentación se complementa con la nivelación topográfica de los postes de catenaria a cuyo efecto se les han adosado de "regletas" fijas, y que constituyen, en la práctica, la referencia fundamental cara al mantenimiento, por su relación directa con la deformación a la plataforma.

El control de la evolución de asientos en los rellenos así instrumentados, ha de cumplir dos objetivos:

- Uno cualitativo, que tiene relación con la posible aparición de fenómenos especiales que produzcan una desviación respecto a un proceso de deformación normal, es decir, directamente relacionada con el tiempo (p. e. asientos bruscos e importantes en épocas lluviosas, etc).

- Otro cuantitativo, que habrá de permitir obtener la deformación total postconstructiva y la relación real respecto de la constructiva, la cual es conocida, precisamente y de forma especial, en estos rellenos.

El aspecto citado en el primer apartado es el más importante cara al problema del mantenimiento de la línea tanto por su mayor magnitud potencial como por la rapidez con que podría llegar a producirse. En esta tipología de asentamientos se incluyen, por ejemplo, los procesos originados por la migración de finos a través del cuerpo del relleno y los aumentos bruscos de la deformabilidad por el humedecimiento de los materiales, ambos casos relacionados directamente con los períodos de lluvias.

El segundo aspecto, por el contrario, estaría enfocado a la comprobación de la validez de la hipótesis establecida en los proyectos respecto a la deformación total y la ley con que se producen en el tiempo, así como la relación de aquélla respecto de la deformación constructiva, todo ello de evidente utilidad en futuras realizaciones. http://informesdelaconstruccion.revistas.csic.es 


\section{ANÁLISIS COMPARATIVO DE ASIENTOS REALES Y ESTIMADOS}

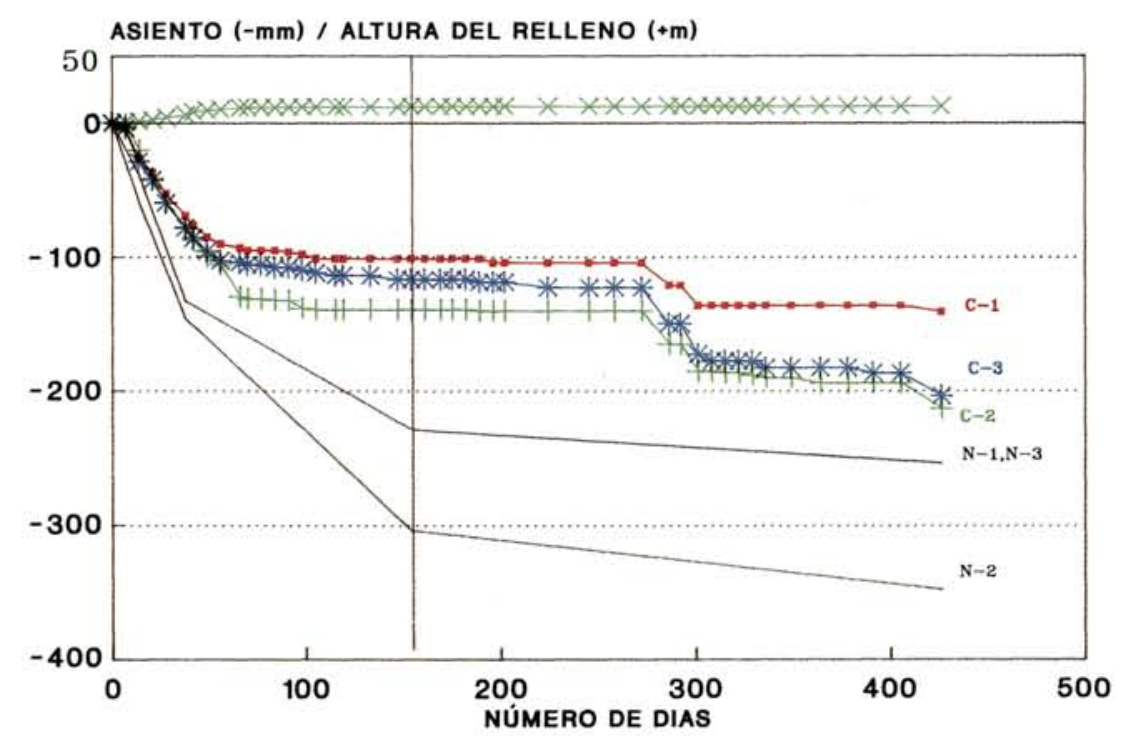

NIVEL SUPERIOR

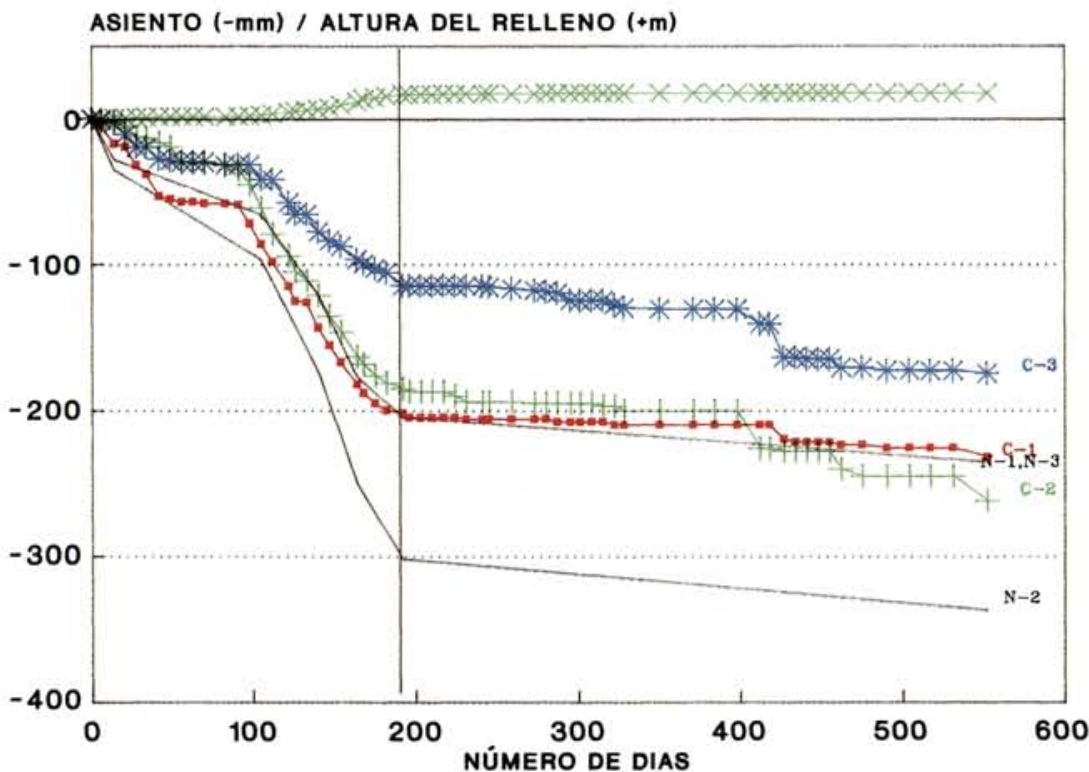

NIVEL MEDIO

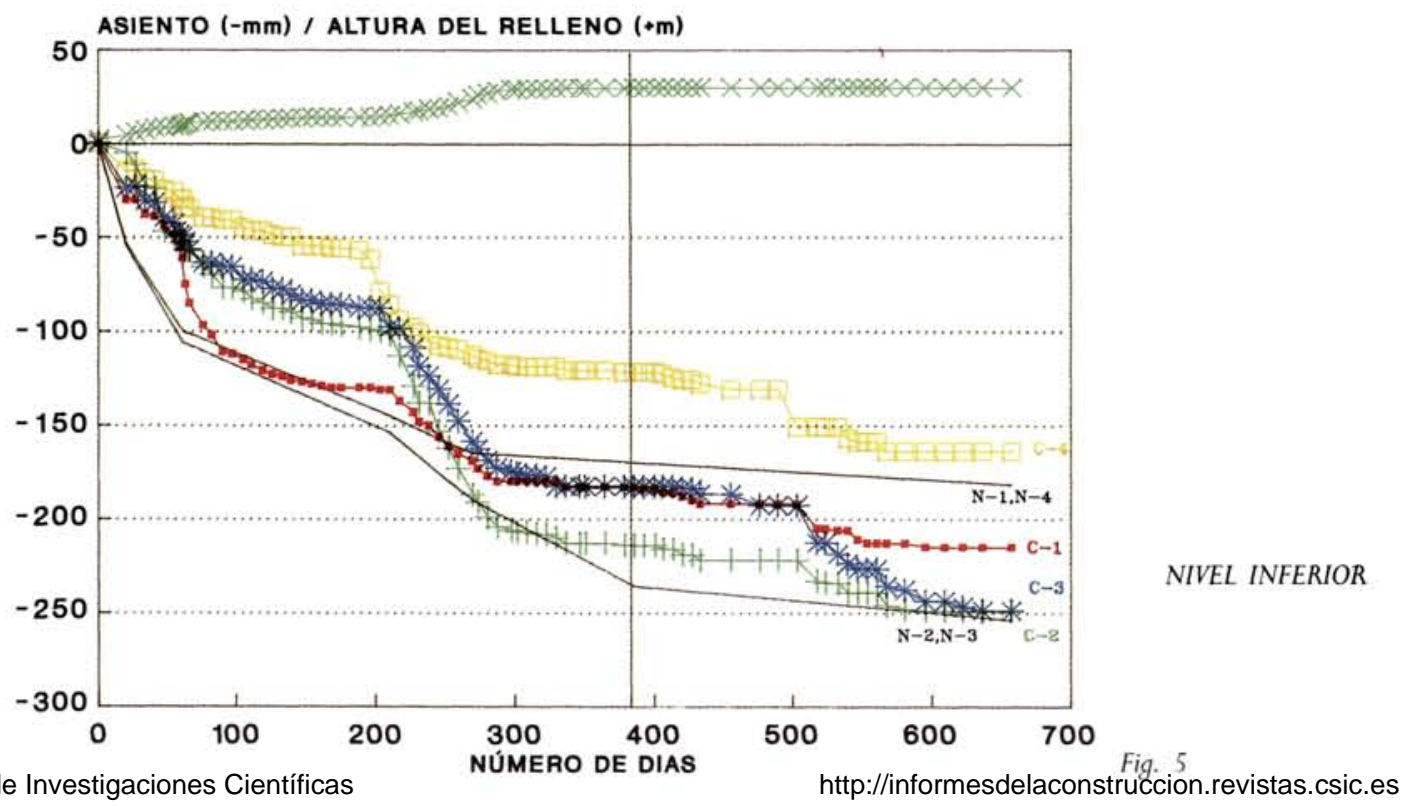




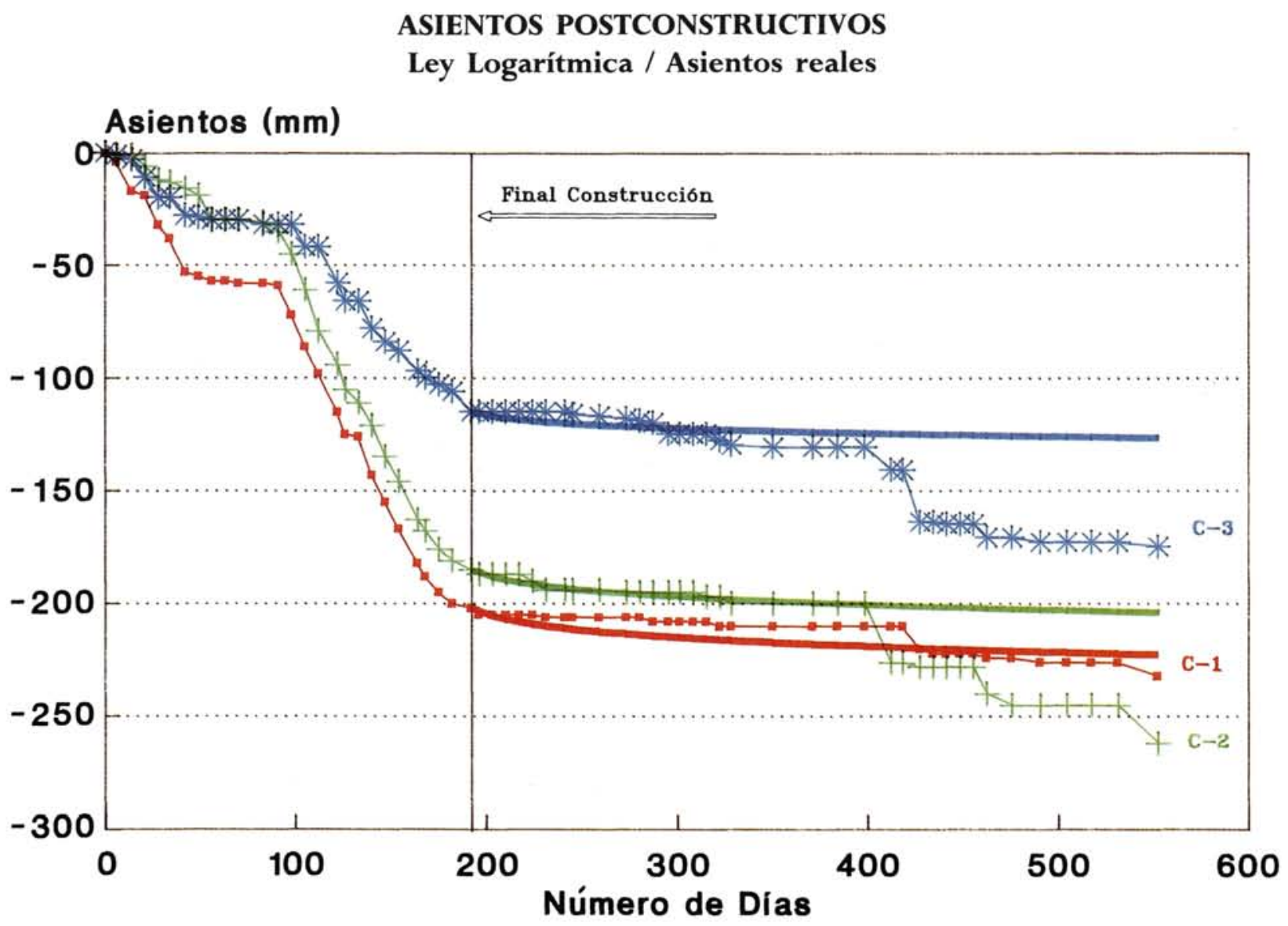

Fig. 6

En la figura 5 se representa el resultado del control de asientos en una sección de relleno instrumentado con tres niveles de células. En estas figuras se diferencia la fase constructiva y la postconstructiva, habiéndose representado tanto los valores medidos como los previstos a partir de los cálculos efectuados mediante un modelo matemático de elementos finitos. Los valores reales obtenidos de la instrumentación se incluyen en las líneas C-1, C-2, etc., representativas de cada una de las células de la sección, siendo los valores teóricos correspondientes los que se señalan con la leyenda $\mathrm{N}-1, \mathrm{~N}-2$, etc.

Como puede comprobarse el ajuste respecto de la curva teórica es, en general, mejor para los niveles inferiores, apreciándose en los superiores el efecto del inicio de la consolidación en las capas más bajas del relleno, lo que es un efecto típico en los rellenos de gran volumen y con amplios plazos de ejecución. En estos casos (c) Consejo superior de Investigaciones Científicas Licencia Creative Commons 3.0 España (by-nc) al asiento constructivo que se produce en las zonas inferiores del relleno por el incremento de las cargas sobre él, se suman parte de los de consolidación postconstructiva del material.

Los asientos registrados durante la construcción han oscilado entre el $1 \%$ y el 3,6\% de la altura del relleno en cada caso, con un valor medio $2,33 \%$, correspondiendo los valores más altos a los rellenos formados con materiales pizarrosos, en los que siempre se han alcanzado valores próximos al $3 \%$.

De acuerdo con estos datos, si se cumple la hipótesis mencionada anteriormente de que los asientos postconstructivos se desarrollarán según una ley logarítmica, dichos asientos alcanzarán valores comprendidos entre un 2 y un 6 por mil de la altura del relleno, produciéndose la mitad de los mismos durante el primer año. 
Si esta hipótesis se cumple, y no aparecen fenómenos atípicos como los mencionados anteriormente, el mantenimiento de la superestructura deberá hacer frente solamente a la mitad de dicho asiento de consolidación, ya que la práctica totalidad de los rellenos se ha puesto en explotación un año después de finalizar la construcción.

En la figura 6 se incluye la representación gráfica de la progresión teórica esperada para los asientos correspondientes a un determinado nivel de células, los cuales se obtienen a partir del ajuste de una ley logarítmica del tipo:

$$
S_{t}=0,2 S_{c} K(\log n)^{a}
$$

siendo:

$S_{t}=$ Asiento constructivo en el momento t.

$\mathrm{S}_{\mathrm{c}}=$ Asiento constructivo.

$\mathrm{n}=$ Número de días transcurridos en el momento $\mathrm{t}$, desde el final de la construcción del relleno.

$\mathrm{K}=0,0758$.

$\mathrm{a}=2,03$.
En el caso representado en esta figura los asientos reales corresponden a las líneas con marcas y los esperados a las de trazo grueso y sin marcas, pudiéndose apreciar la existencia de algunas desviaciones respecto de la evolución teórica obtenida con una ley como la mencionada anteriormente.

En cuanto al seguimiento topográfico de las "cuñas de transición" se han materializado sobre la plataforma itinerarios de referencias fijas de piquetes dotados de "regletas" fijas para la nivelación, en una serie de obras a lo largo de toda la línea, las cuales han sido seleccionadas con el objetivo de muestrear la diferente problemática y tipología, de entre las de mayores alturas.

El sistema topográfico dispuesto en todos los casos consta de referencias cada $5 \mathrm{~m}$, arrancando desde el estribo en el caso de los viaductos y puentes, o cruzando la estructura cuando se trata de una obra de fábrica.

La longitud de los itinerarios cubre siempre la totalidad de la zona ocupada por los distintos elementos que forman las cuñas, llegando hasta la sección en la que se afecta exclusivamente al relleno general.

\section{publicación del ICCET/CSIC}

\section{ACUEDUCTOS ROMANOS EN ESPAÑA \\ Carlos Fernández Casado}

Prof. Dr. Ing. de Caminos, Canales y Puertos

Esta publicación se compone de una serie de articulos, publicados en la Revista "Informes de la Construcciónn, en los cuales se hace un análisis de los acueductos romanos que existen en España y el balance de las condiciones de conservación en que se encuentra cada uno de ellos, incluyendo referencias históricas y literarias. Se ha ilustrado con la reproducción de la valiosa documentación gráfica que posee el prestigioso autor.

Un volumen encuadernado en couché, a dos colores, de $21 \times 27$ centimetros, compuesto de 238 páginas, numerosos grabados, dibujos, fotos en blanco y negro y figuras de linea.

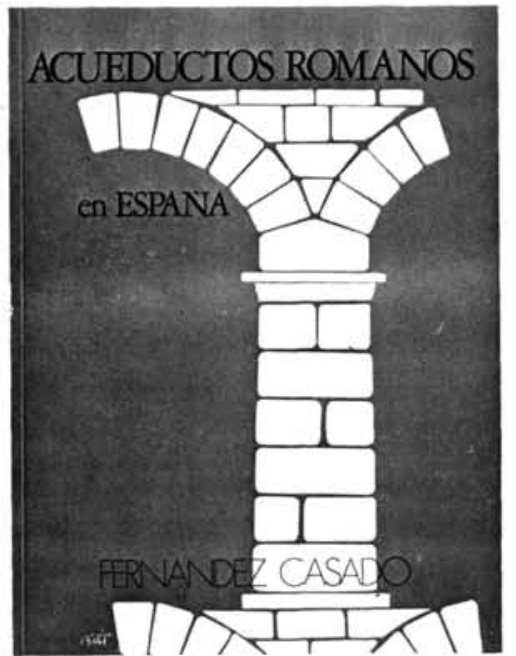

